> Créer un écosystème propice à l'émergence d'initiatives et d'innovations à même de positionner la France comme leader dans l'économie et la société de la connaissance, telle est l'ambition des pôles de compétitivité. À la pointe de l'innovation dans le domaine de la recherche biomédicale et du médicament, le pôle de compétitivité Medicen Paris Région, comprenant plus de 120 partenaires, a été lancé il y a 2 ans. Avec Lyon Biopôle, il fait partie des 2 pôles de compétitivité «à vocation mondiale » dévolus aux Sciences du vivant. Au cœur de Paris Région, première région économique en Europe, ce pôle, constitué autour de 3 domaines thérapeutiques, le cancer, les maladies du système nerveux et les maladies infectieuses, et de 3 thèmes transversaux, l'imagerie médicale, la médecine moléculaire et cellulaire, et le médicament, bénéficie d'atouts exceptionnels. Fin 2006, le pôle Medicen avait labellisé 13 projets, dont le projet TransAl dans l'axe neuroscience, fruit d'une collaboration étroite entre Sanofi-Aventis, Servier et le Commissariat à l'Énergie Atomique (CEA) centrée sur la maladie d'Alzheimer. L'ambition du pôle, en renforçant les interactions et les synergies entre chercheurs, entrepreneurs, universitaires est de contribuer à dynamiser la région île-de-France pour qu'elle devienne à I'horizon 2010 un pôle industriel européen de pointe dans l'innovation thérapeutique. <

\section{Medicen Paris Région, pôle de compétitivité mondial et recherche en neuroscience}

Emmanuel Canet

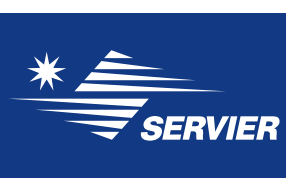

ses, et les disciplines, et à favoriser les interfaces entre la recherche fondamentale et la recherche clinique, publiques ou privées. Leur constitution vise donc à créer les conditions favorables au renforcement ou à l'émergence de nouvelles activités à forte visibilité internationale, compétitives et attractives. Cette stratégie (axe prioritaire de la nouvelle politique industrielle gouvernementale) lancée en septembre 2004 consiste à favoriser, dans une même zone géographique, dans une même dominante sectorielle sur le plan industriel, le développement et la concentration des synergies, des moyens et des savoir-faire complémentaires aux fins d'atteindre la taille critique permettant d'accroître notre potentiel d'innovation et de renforcer notre compétitivité internationale. Le développement des pôles est un des éléments contributifs aux objectifs fixés par les sommets européens de Lisbonne et de Göteborg. C'est dans cette logique que l'articulation avec les politiques communautaires sera systématiquement recherchée. Fondés sur le caractère structurant de l'industrie française et sur son effet d'entraînement sur l'ensemble de l'économie nationale les pôles ont pour vocation à : - être bâtis autour d'un diagnostic partagé des atouts d'un territoire et d'une stratégie commune de développement.

- reposer sur un projet de développement basé sur une forte synergie entre la R\&D et l'activité industrielle proprement dite.

- mettre en réseau, sur une base de proximité géographique, les chercheurs des entreprises et des organismes publiques concernés par une thématique scientifique et/ou technologique donnée.

- faciliter la mutualisation d'un certain nombre de fonctions. 
Sur le plan de la recherche biomédicale, leur constitution devrait permettre au sein d'ensembles de taille critique élevée de faire émerger :

- des innovations technologiques, scientifiques et médicales via des pratiques ancrées sur des allerretour entre recherche fondamentale, recherche finalisée et recherche clinique.

- des innovations organisationnelles concernant la mise en réseau des équipes de recherche publique et des entreprises afin de capitaliser sur les expertises et l'excellence qu'elles concentrent.

De plus, cette forme d'organisation, qui a fait ses preuves dans d'autres pays où elle est parfois très activement soutenue par les pouvoirs publics, répond à deux préoccupations essentielles:

- accroître le potentiel de croissance de l'économie, gage de la préservation de notre compétitivité et de nos équilibres économiques et sociaux.

- maintenir nos compétences médicales scientifiques et technologiques au meilleur niveau afin de jouer un rôle de premier plan dans le progrès thérapeutique et médical.

L'industrie pharmaceutique est de longue date une composante importante du dynamisme technologique, scientifique, économique de notre pays. Le dynamisme futur de l'industrie pharmaceutique en France dépendra de notre capacité à y maintenir une recherche d'excellence dédiée au progrès thérapeutique. La recherche, moteur de cette industrie se caractérise par un accroissement des coûts, une sophistication accrue des technologies et une complexification des questions scientifiques et médicales à résoudre afin de répondre à cette exigence de progrès thérapeutique. Le budget de la recherche pharmaceutique qui en France se situe derrière le Royaume-Uni mais à égalité avec l'Allemagne, représente $14 \%$ des dépenses de recherche de l'industrie en France. L'industrie du médicament emploie directement 100000 personnes en France et la tendance est restée positive jusqu'à maintenant, de même que pour la qualification des emplois. En ajoutant les emplois en amont (chimie fine), en aval (distribution) et tous ceux induits par le secteur, on peut estimer que le médicament emploie en France plus de 300000 personnes.

À terme, le développement de l'industrie pharmaceutique en France est confronté à un double enjeu: d'abord industriel, avec la concurrence croissante à prévoir des pays émergents en particulier en Asie, mais aussi scientifique. En effet, si l'industrie pharmaceutique contribue aux activités de recherche dans le domaine biomédical sur le territoire national, elle le fera d'autant plus que l'innovation sera reconnue et donc valorisée, et qu'elle trouvera à ses côtés une recherche institutionnelle performante.

C'est en ce sens que le groupe de recherche Servier a d'emblée défendu la mise en place de pôles de compétitivité puissants mais limités en nombre; l'idée étant de fédérer, et de focaliser les efforts de recherche, d'instaurer des politiques structurantes et de donner des moyens à la recherche publique lui permettant de se développer et d'être compétitive, et ce au côté d'une recherche industrielle dynamique, créative et performante. L'objectif partagé étant de contribuer au progrès médical et thérapeutique.

\section{Medicen Paris Région, pôle de compétitivité mondial}

Les pôles de très haute technologie, de visibilité mondiale doivent, compte tenu de leur ambition et de la nécessité d'y concentrer expertises et ressources, être limités en nombre. Medicen est l'un des 6 pôles labellisés en juillet 2005 ayant cette ambition. Avec Lyon biopôle, il fait partie des 2 pôles de compétitivité mondiaux dévolus aux sciences du vivant. Localisé en Ile-de-France, il concentre ses activités autour de la recherche biomédicale et du médicament. En 2006, Medicen regroupe plus de 120 partenaires répartis en quatre collèges, les grandes entreprises, les PME et jeunes entreprises innovantes, les organismes de recherche publique (établissements hospitaliers - établissements d'enseignement supérieur et de recherche) - et les collectivités territoriales. Il s'organise dans le cadre d'une association régie par la loi du $1^{\text {er }}$ juillet 1901. Ces pôles s'articulent autour d'un ou plusieurs projets coopératifs de recherche et de développement, l'objectif étant de déboucher, à plus ou moins brève échéance, sur une innovation génératrice de progrès et de valeur économique. La mise en réseau des parties prenantes du pôle appelle un niveau de collaboration élevé, qui ne résulte pas de la simple juxtaposition d'acteurs et d'initiatives, mais bien de la participation au développement d'un projet commun, bâti sur un diagnostic partagé des atouts et des ressources d'un territoire, d'un secteur d'activité et des partenaires concernés. L'implication des collectivités territoriales aujourd'hui reconnue et effective, constitue un élément déterminant de la crédibilité et du succès d'un projet de pôle. Les collectivités, à commencer par les régions, sont partie prenante de la structure chargée de piloter l'élaboration et le suivi du projet de pôle.

La création du pôle Medicen Paris Région se justifie d'autant plus qu'aucune entreprise, et probablement aucun organisme de recherche, ne dispose de l'ensemble des ressources médicales, scientifiques et technologiques qui lui sont nécessaires pour mener à bien des projets complexes et à haute valeur ajoutée dans le domaine de la recherche biomédicale et du progrès thérapeutique. Au cœur de Paris Région, première région économique en Europe, ce pôle bénéficie d'atouts exceptionnels: première région européenne par le nombre d'entreprises du secteur pharmacie, deuxième rang européen par le nombre d'acteurs dans le secteur de l'équipement médical, troisième rang par le nombre d'entreprises de biotechnologies, la présence de plus de $40 \%$ de la recherche publique française dans le domaine de la santé et du plus grand réseau hospitalier européen AP-HP. 
Si le groupe de recherche Servier s'est beaucoup impliqué auprès des autres partenaires du pôle dans la constitution et l'émergence du pôle francilien Medicen, l'objectif est désormais de faire en sorte que ce pôle devienne une réalité. Les différents acteurs doivent comprendre que l'objectif n'est pas simplement de répondre aux appels d'offre et de récupérer tel ou tel financement, autrement dit de bénéficier «d'un effet d'aubaine», mais bien d'avoir une logique de pôle contributive à l'excellence de la recherche française et au progrès médical et thérapeutique. II devient impératif d’y intégrer plus fortement les sciences de la matière. Par exemple au moment où le projet «Soleil» est en état de débuter ses activités, aucun projet à l'interface «Chimie-Biologie» ne semble avoir émergé, c'est pourtant essentiel dans la découverte de nouveaux candidats médicaments à forte valeur ajoutée sur le plan médical. Le pôle santé Ile-de-France peut devenir numéro un européen dans le domaine de la recherche biomédicale et du médicament si chacun des acteurs du pôle reste engagé et tenace quant à la philosophie et aux objectifs. Cela ne se fera pas en quelques mois, mais plutôt en quelques années. Ceci nécessite par ailleurs de réformer certaines pratiques et de faire évoluer les états d'esprit afin d'assurer la flexibilité et la réactivité nécessaire dans un domaine où la compétitivité est extrêmement forte.

Le pôle s'est constitué autour de trois thèmes dits «verticaux», le cancer, s'appuyant sur le cancéropôle lle-de-France, les maladies du système nerveux central et de la moelle en y intégrant les maladies de la vision, et les maladies infectieuses. S'y ajoutent trois thèmes dits «horizontaux», l'imagerie médicale, la médecine moléculaire et cellulaire et le médicament.

L'ambition affirmée des porteurs du projet est que Medicen devienne à l'horizon 2010 le premier pôle industriel européen dans le domaine de l'innovation thérapeutique.

Cette ambition répond à trois enjeux majeurs: un enjeu scientifique pour faire face aux futures révolutions scientifiques et technologiques qui se préparent, en particulier dans les sciences du vivant; un enjeu sociétal, pour améliorer la qualité de vie des hommes, la prédiction et le traitement de maladies; mais aussi et surtout un enjeu économique avec la création d'emplois et de richesse économique. Toutes les thématiques du pôle relèvent de cette triple approche.

Medicen doit plus précisément:

- contribuer à renforcer l'attractivité de l'île-de-France.

- permettre au secteur national des biotechnologies dédiées au médicament de se développer.

- dynamiser la recherche clinique francilienne.

Sept objectifs stratégiques ont ainsi été identifiés :

- renforcer le tissu industriel,

- développer durablement le socle scientifique public et privé,

- faciliter le développement de partenariats étroits entre industriels et la recherche publique,

- contribuer à la formation de chercheurs de haut niveau,

- améliorer les conditions qui garantissent le succès du financement des projets,

- assurer au pôle une forte visibilité internationale.
À ce jour, 13 projets collaboratifs dans le cadre des appels à projets lancés par le fond de compétitivité des entreprises ont été labellisés en 2005 et 2006, pour un montant total de 71 millions d'euros financés à plus de $40 \%$ par les aides publiques.

\section{L’axe neuroscience au sein du pôle Medicen}

Le traitement des maladies du système nerveux constitue un enjeu sociétal majeur du XXI siècle. Ces affections qui concernent le cerveau, la moelle épinière et les nerfs, sont source d'un important problème de santé publique psychiatrique (dépression, psychoses...) ou neurologique (maladies neurodégénératives, épilepsie, accidents vasculaires cérébraux...). Ces pathologies affectent plusieurs dizaines de millions de personnes en Europe et leur fréquence augmente, en raison de l'augmentation de l'espérance de vie. La plupart de ces pathologies ne bénéficient pas de traitement curatif.

L'axe neuroscience du pôle a pour ambition de réunir recherche publique et industrielle et d'être l'un des principaux pôles internationaux dans le domaine de la R\&D des pathologies du système nerveux central. L'excellence scientifique et médicale des équipes constituant le neuropole francilien est comparable en qualité à celle des meilleurs campus américains ou japonais avec les équipes de recherche reconnues internationalement au sein de l'Inserm, du CNRS, du CEA, de l'École normale supérieure, du Collège de France, de l'Université Pierre et Marie Curie, de l'AP-HP et plus de 5000 chercheurs, ingénieurs, doctorants, post-docs et techniciens dans la recherche publique et plus de $60000 \mathrm{~m}^{2}$ de laboratoires, la présence des centres de R\&D dédiés aux neurosciences de Sanofi-Aventis, Servier, Ipsen et la présence d'une vingtaine de sociétés de biotechnologies.

Les actions menées dans le cadre de l'axe neuroscience du pôle Medicen seront de contribuer à faire du neuropole francilien le centre de référence européen en recherche fondamentale, recherche clinique et recherche industrielle dans le domaine des thérapies nouvelles destinées au traitement des maladies du système nerveux et de l'œil, et ce en cohérence avec la création de l'Institut du cerveau et de la moelle (ICM) et de l'Institut de la vision. De plus le CEA (service hospitalier F. Joliot) à Orsay et l'IFR 49 en imagerie neurofonctionnelle offre un plateau technique d'imagerie cérébrale unique en Europe. Ce plateau sera complété par les plateformes Neurospin (neuro-imagerie RMN à champ intense, préclinique et clinique) sur le plateau de Saclay et Mircen (imagerie préclinique multimodale) à Fontenay aux roses. 
Deux projets labellisés ont émergé à ce jour dans le domaine des pathologies du système nerveux au sein de Medicen

- D’une part, le projet Rétinopathies labellisé en 2005 piloté par Mauna Kea Technologies et qui fédère des équipes de recherche de I'Institut de la vision autour d'autres industriels, de l'ESPCI et du CEA.

- D'autre part, le projet transAl, partenariat établi avec Sanofi-Aventis et Servier et le CEA dans le domaine des maladies neurodégénératives.

La recherche de médicaments capables de modifier la progression de la maladie d'Alzheimer représente une priorité pour la recherche biomédicale compte tenu de l'ampleur du besoin médical et de la prévalence croissante de la maladie. De nombreux chercheurs, issus de la recherche publique, du CEA ou d'équipes de recherche industrielle telles que Servier ou Sanofi-Aventis, sont résolument engagés dans cette recherche. Le projet TransAl dont l'objectif est de contribuer à relever ce défi a été labellisé par Medicen en 2006 . C'est le tout premier projet collaboratif du pôle Medicen dédié aux pathologies du système nerveux central. Ce projet a pour objectif de développer un modèle expérimental chez le primate permettant d'évaluer les mécanismes impliqués dans la genèse et la progression de la maladie d'Alzheimer, et d'identifier des biomarqueurs pertinents afin non seulement de mieux diagnostiquer la maladie mais également de mieux évaluer le potentiel thérapeutique de nouveaux candidats médicaments. La maladie d'Alzheimer est une affection dégénérative (caractérisée par une mort cellulaire lente prématurée) du système nerveux central, dont la fréquence augmente avec le temps en raison de l'augmentation de l'espérance de vie (actuellement 600000 personnes atteintes en France). Elle est caractérisée par la présence de difficultés intellectuelles qui s'aggravent progressivement, débutant par des troubles de mémoire sur les faits récents puis anciens. Aujourd'hui le diagnostic est le plus souvent tardif et ne peut être établi avec certitude qu'à partir d'une étude neuropathologique post-mortem du cerveau. L'examen neuropathologique doit révéler la présence de dépôts extracellulaires (plaques séniles) constitués de peptides $\beta$-amyloïdes ( $A \beta$ ) agrégés et d'altérations du cytosquelette sous la forme d'agrégats intracellulaires de protéines Tau hyperphosphorylées. Cette difficulté d'établir un diagnostic précoce est associée à l'absence de traitement médicamenteux susceptible de ralentir la progression de la maladie ou d'agir sur les mécanismes physiopathologiques responsables de la maladie. Mieux comprendre les causes de la maladie en associant recherche clinique et recherche expérimentale sur des modèles pertinents, identifier des marqueurs précoces de la maladie ou des moyens permettant d'évaluer l'évolutivité de la maladie pourrait contribuer à ouvrir de nouvelles perspectives et améliorer ainsi la prise en charge de ces malades.

De nombreux modèles (spontanés, chirurgicaux ou transgéniques) ont été développés chez la souris mais aucun de ces modèles ne parvient à reproduire la symptomatologie ni l'ensemble des lésions anatomopathologiques de la maladie. Les modèles plus étudiés reposent sur la surexpression de gènes humains mutés associés aux formes familiales, rares, de la maladie (codant la protéine précurseur du peptide $A \beta$ qui s'accumule dans les plaques séniles observées chez les patients) ou encore sur la surexpression de la protéine Tau hyperphosphorylée. Certains primates sont susceptibles, au cours d'un processus de vieillissement normal, de produire spontanément des lésions similaires à celles observées chez l'humain: plaques séniles et dégénérescence neurofibrillaire, s'accompagnant d'altérations comportementales, notamment cognitives. Dans le projet TransAl il est envisagé de créer un modèle expérimental chez le primate (macaque) en induisant une accumulation intracérébrale de peptide $A \beta$, une des causes supposées de la maladie d'Alzheimer selon une stratégie différente de celle utilisée pour les modèles murins transgéniques. Cette stratégie consiste à générer un déficit de clairance du peptide $A \beta$, processus plus proche de ce qui est observé dans les formes sporadiques de la maladie. L'expertise scientifique et technique du CEA, que ce soit dans le domaine du transfert somatique de gènes par vectorisation virale intracérébrale ou de l'imagerie cérébrale, sera critique quant à la mise en œuvre et à la réussite de ce programme.

Le second objectif du projet TransAl concerne l'identification, la caractérisation et la validation de marqueurs pour l'imagerie (IRM, TEP-atrophie, marqueurs de plaques, imagerie fonctionnelle...) ou de biomarqueurs (approche protéomique à partir du LCR). Les études sont focalisées sur les biomarqueurs actuellement utilisés chez l'homme, mais aussi sur des biomarqueurs spécifiques des différents stades de la maladie et pouvant être détectés par les techniques d'imagerie médicale. De tels biomarqueurs, validés et pertinents, devraient permettre d'identifier les populations de patients plus homogènes et les plus à même de bénéficier des nouvelles approches thérapeutiques en cours de mise au point, d'évaluer plus rapidement l'évolutivité de la maladie, l'efficacité des candidats médicaments et d'accélérer ainsi la mise à disposition de traitements innovants adaptés aux différentes formes ou stades d'évolution de la maladie d'Alzheimer.

Dans le cadre des projets structurants du pôle, le projet de création d'un centre de ressources biologiques humaines (banque d'ADN, banque de tissus) dédié aux maladies neurologiques (Alzheimer, Parkinson...) et psychiatriques (schizophrénie, troubles bipolaires) est en cours d'élaboration et pourrait voir le jour d'ici fin 2007. Outre la contribution à une recherche à visée cognitive performante, ce projet contribuerait également à identifier et valider des cibles thérapeutiques nouvelles dans les domaines concernés et à valider des marqueurs d'intérêt (diagnostic, pronostic...). Fédérer ces ressources, s'assurer de leur traçabilité, de leur qualité et de la qualité des informations qui leur sont associées et ce dans le respect des lois de bioéthique, 
les rendre accessibles dans le cadre de programmes de recherche bien identifiés en constitue les objectifs et un enjeu capital pour la recherche en neuroscience en Île-de-France, publique ou privée.

Donner à la recherche les moyens de relever le défi du progrès thérapeutique dans des pathologies où les besoins sont majeurs, en s'organisant autrement, en assurant le continuum entre recherche fondamentale et recherche finalisée, entre recherche publique, jeunes entreprises et industries de santé, telle est l'ambition renouvelée de Medicen Paris Région. $\diamond$

\section{SUMMARY}

Medicen: A world-class «competitiveness cluster》

in the Paris region incorporating a neuroscience « subcluster 》

The French public-private partnerships known as "competitive clusters" [pôles de compétitivité $(\mathrm{PdC})$ ] are intented to be novel and ambitious engines of regional growth, employment and biomedical innovation. Partly funded by government and local councils, they aim to capitalize on regional expertise by bringing together basic scientists, clinicians, innovative entrepreneurs and local decision-makers around specific themes that have become too costly and complex for any of these actors to tackle alone. Clusters provide the critical mass required both to underpin innovation potential and to authenticate regional claims to international competitiveness. Medicen is a biomedicine and therapeutics cluster comprising 120 partners from four broad "colleges" in the greater Paris region: major industry, small and medium-sized businesses, teaching hospitals/State research bodies, and local councils. Chief among its cooperative R\&D projects is the neuroscience subcluster, in which "TransAl" the neurodegenerative disease project, counts Sanofi-Aventis, Servier and the French Atomic Energy Commission [Commissariat à l'Énergie Atomique (CદA)] as key partners. One main aim is to develop an experimental model in rhesus monkeys in which a putative cause of Alzheimer's disease, intracerebral accumulation of $\beta$-amyloid peptide, is generated by impairing the peptide's clearance. The other aim, in which the nuclear medicine expertise of the CEA will be crucial, is to identify, characterize and validate markers for magnetic resonance and positron emission tomography imaging, and to source biomarkers from cerebrospinal fluid proteomics. A human biological resource centre (DNA and tissue banks) project dedicated to neurological and psychiatric disease should be up and running in 2007. Only through fundamental restructuring of resources on such a large cooperative scale are solutions likely to be found to the major problems of modern medicine, bringing healthcare and regional socioeconomic benefits in its wake. $\diamond$

\section{REMERCIEMENTS}

Je tiens à remercier le Professeur Yves Agid, responsable de la thématique Neuroscience du pôle Medicen pour la relecture critique de cet article.

\section{BIBLIOGRAPHIE}

1. Pour un écosystème de la croissance. Paris : Rapport au premier ministre, Blanc C. 2005.

2. www.medicen.org

3. Dhenain M, Lehéricy S, Duyckaerts C. Le diagnostic : de la neuropathologie à l'imagerie cérébrale. Med Sci (Paris) 2002 ; 18 : 697-708

4. Hardy J, Duff K, Hardy K, et al. Genetic dissection of Alzheimer's disease and related dementias: amyloid and its relationship to tau. Nat Neurosci $1998 ; 1: 355-8$.

5. Poduslo JF, Curran GL, Peterson JA, et al. Design and chemical synthesis of a magnetic resonance contrast agent with enhanced in vitro binding, high blood-brain barrier permeability, and in vivo targeting to Alzheimer's disease amyloid plaques. Biochemistry 2004 ; 43 : 6064-75.

6. Higuchi M, Iwata N, Matsuba Y, et al. (19)F and (1)H MRI detection of amyloid beta plaques in vivo. Nat Neurosci $2005 ; 8$ : 527-33.

7. Blennow K, Hampel H. CSF markers for incipient Alzheimer's disease. Lancet Neurol $2003 ; 2: 605-13$.

8. Andreasen N, Blennow K. CSF biomarkers for mild cognitive impairment and early Alzheimer's disease. Clin Neurol Neurosurg 2005; $1073: 165-73$.

9. Delatour B, Le Cudennec C, દl Tannir-દl Tayara N, Dhenain M. Transgenic models of Alzheimer's pathology: success and cavaet. In : Progress in Alzheimer's disease research. Paris : Éditions Nova, 2007 (sous presse).

10. Gearing M, Rebeck GW, Hyman BT, et al. Neuropathology and apolipoprotein $\varepsilon$ profile of aged chimpanzees: implications for Alzheimer disease. Proc Natl Acad Sci USA 1994 ; 91 : 9382-6.

11. Schultz C, Hubbard GB, Rub U, Braak H. Age-related progression of tau pathology in brains of baboons. Neurobiol Aging 2000; $21: 905-12$.

12. Deglon N, Hantraye P. Viral vectors as tools to model and treat neurodegenerative disorders. J Gene Med 2005 ; 7 : 530-9.

13. Albert M, DeCarli C, DeKosky S, et al. The use of MRI and PET for clinical diagnosis of dementia and investigation of cognitive impairment: a consensus report. Report of the Neuroimaging Work Group of the Alzheimer's Association, 2005.

\section{TIRÉS À PART}

$\varepsilon$. Canet 